INPLASY

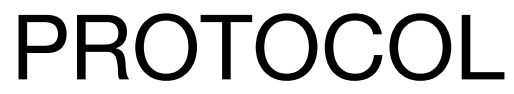

To cite: Kouloutbani et al. Effects of physical exercise on the neuropsychiatric symptoms of patients with dementia: A protocol of systematic review and metaanalysis. Inplasy protocol 202160108. doi: 10.37766/inplasy2021.6.0108

Received: 28 June 2021

Published: 28 June 2021

Corresponding author: Komanthi Kouloutbani

komanthi@gmail.com

Author Affiliation:

School of Physical Education and Sport Science, National and Kapodistrian University of Athens.

Support: No financial support.

Review Stage at time of this submission: Risk of bias assessment.

Conflicts of interest: None declared.

\section{Effects of physical exercise on the neuropsychiatric symptoms of patients with dementia: A protocol of systematic review and meta-analysis}

Kouloutbani, K; Venetsanou, F2; Dinas, P3; Karteroliotis, K4; Politis, $A^{5}$.

Review question / Objective: The purpose of this systematic review and meta-analysis is to evaluate the effects of physical exercise on the neuropsychiatric symptoms of dementia patients.

Condition being studied: In recent years, the percentage of people with mild cognitive impairment $(\mathrm{MCl})$ or dementia is constantly increasing. A major concern in dementia treatment is the management of the neuropsychiatric symptoms that are common in this population. Neuropsychiatric symptoms, such as agitation, apathy, anxiety, depression, are essential elements of the phenomenology of dementia, and their mismanagement has a multifaceted impact on both patients and their caregivers. Structured physical activity is increasingly recognized as an effective non-pharmacological treatment of neuropsychiatric symptoms. However, findings from previous systematic reviews and meta-analyses do not provide clear picture on this important issue.

INPLASY registration number: This protocol was registered with the International Platform of Registered Systematic Review and Meta-Analysis Protocols (INPLASY) on 28 June 2021 and was last updated on 28 June 2021 (registration number INPLASY202160108).

\title{
INTRODUCTION
}

Review question / Objective: The purpose of this systematic review and meta-analysis is to evaluate the effects of physical exercise on the neuropsychiatric symptoms of dementia patients.
Rationale: The management of neuropsychiatric symptoms is a major challenge in the treatment of dementia. Since no effective therapy has been found to date and drug treatments may be associated with significant side effects, the 
need for alternative non-pharmacological interventions is imperative.

Condition being studied: In recent years, the percentage of people with mild cognitive impairment $(\mathrm{MCl})$ or dementia is constantly increasing. A major concern in dementia treatment is the management of the neuropsychiatric symptoms that are common in this population. Neuropsychiatric symptoms, such as agitation, apathy, anxiety, depression, are essential elements of the phenomenology of dementia, and their mismanagement has a multifaceted impact on both patients and their caregivers. Structured physical activity is increasingly recognized as an effective non-pharmacological treatment of neuropsychiatric symptoms. However, findings from previous systematic reviews and meta-analyses do not provide clear picture on this important issue.

\section{METHODS}

Search strategy: The search was conducted using the following electronic d a t abases: SCOPUS, PubMed, SPORTDiscus, Web of science and ScienceDirect. Wherever possible, terms, such as: "physical activity", "exercise", "Alzheimer's disease" "dementia", "mild cognitive impairment", "neuropsychiatric symptoms", from the MEdical Subject Headings (MESH) or keywords were used applying the appropriate algorithms.

Participant or population: Institutionalized or non-institutionalized patients with $\mathrm{MCl}$ or dementia. Studies involving model organisms were excluded.

Intervention: Implementation of physical exercise programs with reference to its specific characteristics such as type, frequency, intensity and duration.

Comparator: The effect of exercise will be studied through the comparison of exercise interventions to non-exercise interventions (exercise vs usual treatment).

Study designs to be included: We only considered randomized controlled trials.
Eligibility criteria: The inclusion criteria, based on the PICOS method (Participants, Intervention, Comparison, Outcomes, Studies) were the following a) Participants: institutionalized or non-institutionalized patients with MCl or dementia, b) Intervention: implementation of physical exercise programs, c) Comparison: experimental group vs control group, d) Outcomes: The effect of physical exercise in neuropsychiatric symptoms of dementia and e) Type of studies: randomized controlled trials.

Information sources: Five different databases were used, i.e., "Scopus", "PubMed", "SPORTDiscus", "Web of Science" and "ScienceDirect".

Main outcome(s): The 12 domains that comprise the Neuropsychiatric Inventory (NPI) scale, i.e., delusions, hallucinations, aggression, depression, anxiety, euphoria, apathy, disinhibition, irritability, aberrant motor behavior, sleep and night time behavior disorders and appetite/eating changes. It should be noted that studies investigating the above symptoms, using assessment tools other than the NPI, were also included.

Additional outcome(s): Specific neuropsychiatric symptoms are secondary outcomes of the review.

Quality assessment / Risk of bias analysis: The assessment of the risk of bias will be carried out through the "Cochrane Collaboration's tool for assessing the risk of bias", which encompasses the following items: random-sequence generation (selection bias), allocation concealment (selection bias), blinding of participants and personnel (performance bias), blinding of outcome assessment (detection bias), incomplete outcome data (attrition bias), selective reporting (reporting bias), and other sources of bias. The evaluation level of each item includes "low risk" (+), "high risk" (-), and "unclear risk" (?) of bias. The assessment will be conducted by two independent investigators and in the case of disagreement a third experienced 
reviewer will intervene to resolve the issue through discussion and negotiation.

Strategy of data synthesis: The Review Manager (v.5.3.5) software will be used for the analysis of quantitative data (forest plot, heterogeneity analysis and sub-group analysis). The heterogeneity between the included studies will be evaluated using the 12 test. If relative heterogeneity is high (12>50\%), the random effects model will be utilized. If relative heterogeneity is low $(12<50 \%)$, the data will be analyzed using the fixed effects model. The results of the reviewed studies that will not be suitable for the meta-analyses will be discussed, separately.

Subgroup analysis: In the possibility of high heterogeneity across the studies, subgroup analysis will be conducted to explain the potential causes of heterogeneity.

Sensitivity analysis: If necessary, we will also conduct a sensitivity analysis.

Language: English.

Country(ies) involved: Greece.

Keywords: Physical exercise, dementia, Alzheimer's disease, neuropsychiatric symptoms, mild cognitive impairment.

Contributions of each author:

Author 1 - Komanthi Kouloutbani.

Author 2 - Fotini Venetsanou.

Author 3 - Petros Dinas.

Author 4 - Konstantinos Karteroliotis.

Author 5 - Antonios Politis. 